\title{
The Substellar Luminosity and Mass Functions of the Trapezium Cluster Down to the Deuterium Burning Limit
}

\author{
August A. Muench \& Elizabeth A. Lada \\ Department of Astronomy, University of Florida, 211 SSRB, \\ Gainesville, FL 32611, USA \\ Charles J. Lada \\ Harvard-Smithsonian Center for Astrophysics, 60 Garden Street, MS \\ 72, Cambridge, MA 02138, USA
}

João F. Alves

European Southern Observatory, Karl-Schwartzschild-Strasse 2, 8574 Garching Germany

\begin{abstract}
We have employed an advanced suite of Monte Carlo calculations to model the infrared luminosity function (KLF) of the Trapezium cluster and to derive its Sub-stellar Initial Mass Function down to the deuterium burning limit. Independent of the details, we find that substellar objects can account for no more than $\sim 22 \%$ of the total number of likely cluster members. This cluster's substellar IMF steadily declines in a power-law fashion over most of the brown dwarf regime, however the formation of a secondary peak in the cluster's substellar KLF suggests the existence of a corresponding secondary peak in the substellar IMF between 10 and 30 times the mass of Jupiter based upon the seemingly degenerate behavior of the theoretical substellar mass-luminosity relation.
\end{abstract}

\section{Introduction and Models}

With a population of $100-150$ brown dwarf candidates lying within a $0.12 \mathrm{pc}^{2}$ region (Muench et al. 2001), the $\tau \sim 1$ Myrs Trapezium Cluster, which is but the core of the larger Orion Nebula Cluster, has a higher concentration of brown dwarfs than any other known region or cluster, thus providing the necessary statistics to securely identify the form of the substellar initial mass function (IMF). Beginning with Muench, Lada, \& Lada (2000) we have explored this cluster's substellar IMF by constructing and analyzing the cluster's $\mathrm{K}$ band luminosity function (KLF). Using a deeper $\mathrm{JHK}_{\mathrm{S}}$ survey described in Muench et al. (2002) we have extended this study to a volume limited sample sensitive to a $0.01 M_{\odot}$ brown dwarf seen through an extinction of $\mathrm{A}_{V}=9$.

We analyzed the cluster's KLF by employing a Monte Carlo based population synthesis algorithm for pre-main sequence stars first presented in Muench, Lada, \& Lada (2000) and improved in in Muench et al. (2002) to include the effects of extinction and infrared excess. Adopting a star forming history from 


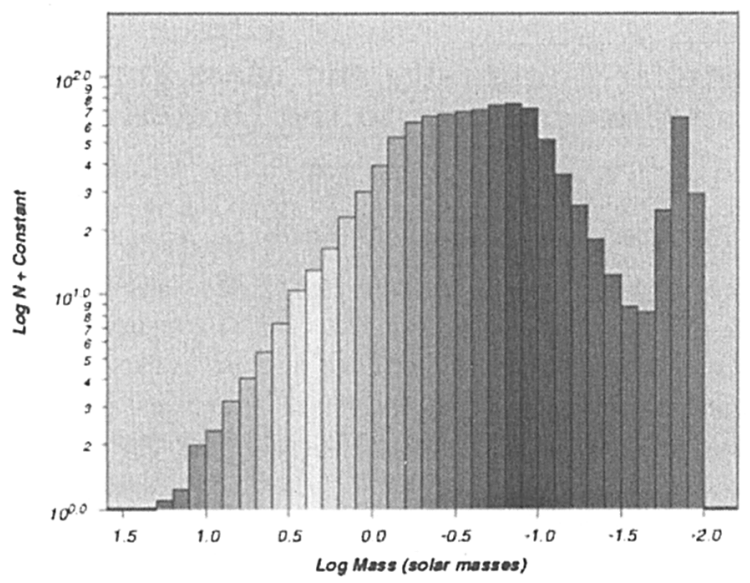

Figure 1. The stellar and substellar IMF for the Trapezium Cluster derived from luminosity function modeling.

Hillenbrand (1997), we constructed a suite of synthetic KLFs having a range of underlying IMFs, which we parameterized as a series of power-laws connected at mass breaks. From fits of these model KLFs to the observed Trapezium KLF we derived the underlying IMF parameters with associated error bars.

\section{Results}

From our fits, we derive a Trapezium IMF (Figure 1) that declines across the hydrogen burning limit and down to $0.02-0.03 M_{\odot}$ with a slope of $\Gamma \sim 0.7$. Independent of our modeling procedure, we find that brown dwarfs can account for no more than one in four of the objects in this cluster. We find that a secondary peak in the cluster's substellar KLF corresponds to a significant secondary IMF peak near the deuterium burning limit based on two different sets of PMS tracks. While this feature may contain as many as $30 \%$ of the brown dwarfs in this cluster, it may also be related to a previously unknown feature in the mass-luminosity relation (see Jameson 2002, this volume). Alternately, the presence of a peak in the Trapezium Cluster's substellar KLF and IMF may also suggest that a secondary physical mechanism is responsible for the formation of very low mass brown dwarfs. Sensitive studies of the properties of disks around these lowest mass sources (for example, Liu or Testi, this volume) may be essential for distinguishing between competing theories.

\section{References}

Hillenbrand, L. A. 1997, AJ, 113, 1733

Muench, A. A., Lada, E. A., \& Lada, C. J. 2000, ApJ, 533, 358

Muench, A. A., Alves, J. F., Lada, C. J., \& Lada, E. A. 2001, ApJ, 558, L51

Muench, A. A., Lada, E. A., Lada, C. J., \& Alves, J. F. 2002, ApJ, 573, 366 